\title{
FORESTALLING GROWTH RATE IN TYPE II DIABETIC PATIENTS USING DATA MINING AND ARTIFICIAL NEURAL NETWORKS: AN INTENSE SURVEY
}

\author{
Kiran Bala Dubey \\ Govt. Nagarjuna Post Graduate College of Science, Raipur, Chhattisgarh, India \\ Dr. Gyanesh Shrivastava \\ School of Information Technology, MATS University, Raipur, Chhattisgarh, India
}

\begin{abstract}
The race for urbanization and thirst for high living status leads to unhealthy life. As the result a rapid growth in number of diabetic patients in urban areas approaching to its deadline. In this situation it become a prime necessity for physicians and health workers to recognize accurate growth rate in number of diabetic patients. Artificial Neural Network is used as one of the artificial intelligent technique for forestalling growth rate of type II diabetic patients. Diabetes occurred due to increased level of glucose in blood. In this paper, an intense survey is done for the prediction of Type II diabetes using different Data Mining tools and Artificial Neural Network techniques, is presented. This survey is aimed to recognize and propose an effective technique for earlier prediction of the Type II diabetes. The data mining techniques like C4.5 Classifier, Support Vector Machine and K-Nearest Neighbour are compared for this work with Artificial Neural Network. As the results Artificial Neural Network found with a great accuracy of $89 \%$.
\end{abstract}

Keywords: Artificial Neural Network, type II Diabetes, Prediction, BPN.

Cite this Article: Kiran Bala Dubey and Dr. Gyanesh Shrivastava, Forestalling Growth Rate in Type II Diabetic Patients using Data Mining and Artificial Neural Networks: An Intense Survey, International Journal of Computer Engineering and Technology, 10(3), 2019, pp. 31-38.

http://iaeme.com/Home/issue/IJCET?Volume=10\&Issue $=3$

\section{INTRODUCTION}

Nowadays diabetes has became the major health problem among the people of all age groups. Diabetes is a non-curable disorder known round the globe. The medicine cost of a diabetic patient is very expensive, which leads to high humanity in developing countries. The detection of disease is a big difficulty. Researchers look the significance of diagnosis and prediction of diabetes. There are 2 category of diabetes - Type I and Type II. The Type I 
diabetes occurs when the pancreas secretes zero or little amount of insulin. It generally happens in childhood or in teenage people and affects $10 \%$ of people. When the pancreas does not secrete sufficient insulin as per the body requirement then ensure diabetes of Type II. It usually happens middle or old ager persons and affects $90 \%$ of people. More than $80 \%$ people die due to other diseases caused by diabetes. Therefore, it is mandatory to diagnose the diabetes as early as possible then it can be curable. There are different techniques for early detection of diabetes.

A simple statistical method cannot be a better way to diagnose Diabetes. With the improvement of information technology, data mining techniques are used to take out the interesting patterns from big data repository. To prevent complications in diabetes, patients have to know the future value of blood glucose so they can take precaution and be normal as possible. In this study, we found that Artificial Neural Network (ANN) is used for an accurate blood glucose level prediction of Type II Diabetes. ANN has mathematically implemented model and is easy to use compared to other statistical methods.

\section{LITERATURE REVIEW}

Kavitha, K. et al., 2012, [1] have presented an approach to design a platform for enhancing efficiency of health supervising using CART method for predilection of diabetes. The proposed system achieved the accuracy and a precision of $96.39 \%$ and $100.00 \%$ in detecting Diabetes.

Singh, S., K. and Archana, 2013, [3] proposed an intelligent and useful methodology for the automatic detection of Diabetes. This methodology was designed using Artificial Neural Network technique and implemented on MATLAB tool. In proposed model, user itself sitting on home can diagnose whether they were suffering from Diabetes or not. They only need to provide some physical parameters to the proposed model then it was detected whether that person was suffering from Diabetes or not.

Rahman, R., M. et al. 2013, [4] compared the different classification techniques using three data mining tools named WEKA, TANAGRA and MATLAB on diabetes data. The result showed that the best algorithm in WEKA was J48graft classifier whose accuracy of $81.33 \%$ that takes 0.135 seconds for training. In TANAGRA, Naïve Bayes classifier provided accuracy of $100 \%$ with training time 0.001 seconds. In MATLAB, ANFIS had $78.79 \%$ accuracy. They concluded that the TANAGRA tool was best as compared to WEKA and MATLAB.

Rajesh, K. and Sangeetha, V. 2012, [5] found the appropriate data mining methods and techniques for classification of diabetes dataset. The result showed that $\mathrm{C} 4.5$ classification algorithm efficiency rate was $91 \%$ than others.

Iyer, A. et al. 2015, [6] observed that mostly women were suffering from Diabetes worldwide. They used Decision Tree and Naïve Bayes classification algorithms and proposed a faster and more effective technique for diagnosing of diabetes for timely treatment of the patients. Experimental results showed the capability of the proposed model.

Thirumal, N., and Nagarajan, 2015, [7] compared various data mining techniques such as Naïve Bayes, Decision trees, k-Nearest neighbour and SVM on PIMA Indian diabetes dataset to predict diabetes. After the experiments they concluded that k-Nearest neighbour provided lower accuracy than other algorithms.

Barakat et al. 2010, [8] proposed support vector machines (SVMs) to diagnose the diabetes. Results on a real-life diabetes dataset showed that the SVMs provided with prediction accuracy of $94 \%$, sensitivity of $93 \%$, and specificity of $94 \%$. 
Forestalling Growth Rate in Type II Diabetic Patients using Data Mining and Artificial Neural Networks: An Intense Survey

In Kumari, M. et al. 2014, [9] paper Bayesian Network classifier was proposed to predict diabetes on dataset collected from a hospital. They used WEKA tool for the experiment and analysis. The accuracy of the model was compared with other algorithms and found Bayesian network classifier showed the best accuracy of 99.51 percent and error was 48 percent.

Priya, S. and Rajalaxmi, R., 2012, [10] developed a Hybrid Prediction Model (HPM) using k-means clustering and C4.5 classifier algorithms. Result on the Pima Indian Diabetes dataset showed that HPM produced an improved accuracy of $97.93 \%$.

Kanungo, Y. et al. 2017, [11] studied that Diabetic Retinopathy (DR) was an eye disease caused mainly due to long-term diabetes that cause blindness in developed nations. Detection of DR symptoms in time can prevent the vision impairment in majority of cases. There has been a need for complete and automated DR detection tool and method. Their experiments showed that the hyper-parameters played a key role in the performance of a model and deriving concrete methods to find the optimal hyper-parameters quickly. In this study colour photography has been used and the results suggest that the model has realistic clinical potential.

Rahimloo, P. and Jafarian, A., 2016, [12] combined the statistical models and neural networks technique to create a new model. After analysis they found that new model has minimum error and maximum reliability.

$\mathrm{Wu}, \mathrm{H}$. et al. 2017, [13] proposed a new model using data mining techniques to predict type 2 diabetes. The model has two parts, the improved K-means algorithm and the logistic regression algorithm. Result on Pima Indians Diabetes Dataset was comparing with other researcher's results. They concluded that the proposed model reached a $3.04 \%$ higher accuracy of prediction than other researchers. As a result, this model was to be useful for the real health management of diabetes.

Motka, R. et al. 2013, [14] used classification systems for medical decision-making in short period. For this they used four different approaches namely Neuro fuzzy inference system, principal component analysis, neural network and machine learning and classify whether subjects have Diabetic or Non-diabetic.

Anjaneya L.H. and Holi M. S., 2016, [15] proposed a new model to consider time domain and frequency domain features of the EMG signals and used neural network to perform the diabetes classification. They used MATLAB tool and showed the accuracy of proposed model is $97.05 \%$.

Karan, O. et al. 2012, [16] presented a client and server two-tier persistent healthcare architecture. The access of neural network operations on client and server sides with wireless network communications between them for real time use of persistent health services.

According to Jasim, I., S. et al. 2017, [17] paper classification has performed by choosing k-nearest neighborhood algorithm and Artificial Neural Network techniques on Pima Indian Diabetes dataset using spiral spinning technique. The results showed that after some change in architecture, performance of Artificial Neural Network gave better results than k-nearest neighborhood.

Santhanam, T. and Padmavathi, M., S., 2014, [18] compared K-Means clustering and Statistical Outlier detection techniques on cleaned datasets and they found that the reduction rate of Outliers was less than K-Means clustering.

Hamdi, T. et al. 2017, [19] proposed an Artificial Neural Network technique for an accurate blood glucose level prediction of Type1 Diabetes. To validate the proposed method, real Continuous Glucose Monitoring data of 12 patients were investigated. Experimental 
results have shown that the proposed model was able to predict blood glucose with minimum errors and can be used to detect hyperglycemia or hypoglycemia 15 minutes in advance.

Geman, O. et al. 2017, [20] proposed a hybrid Adaptive Neuro-Fuzzy Inference System (ANFIS) model for classifying diabetic patients. This model was implemented on ANFIS Fuzzy Logic and MATLAB Toolbox using Pima Indians Diabetes Dataset and proved an accuracy of $85.35 \%$ for training data and $84.27 \%$ for testing data.

NirmalaDevi, M. et al. 2013, [22] presented an amalgam model for classifying Pima Indian diabetic database. This amalgam model combined k-means with k-Nearest Neighbour (KNN) with multi-step pre-processing. In this amalgam model, the qualities of the data were enhanced by removing noisy data and improve the accuracy and efficiency of the KNN algorithm. Experimental results have shown that the proposed model produced best result for different $\mathrm{k}$ values. If $\mathrm{k}$ value is more, the proposed model obtained the classification accuracy of $97.4 \%$.

Swain, A. et al. 2016, [23] proposed a model for prediction and classification of Diabetes using Artificial Neural Network (ANN) and hybrid Adaptive Neuro-Fuzzy Inference System (ANFIS). The model was tested by using the data of 100 persons with mean age of 42 years with an equal proportion of male and female. The result revealed that on the basis of accuracy the ANFIS approach was more acceptable than the ANN approach.

Zecchin, C. et al. 2011, [24] developed a new short-term glucose prediction algorithm based on a neural network. In addition to past continuous glucose monitoring (CGM) readings, they exploit information on carbohydrates intakes that quantitatively described through a physiological model. Results showed that the new method outperforms than other published algorithms. Qualitative preliminary results on a real diabetic subject confirm the potentialities of the new approach.

In M. Durairaj and G. Kalaiselvi, 2015, [26] paper a detailed survey was conducted on the application of different data mining techniques for the prediction of diabetes. This survey was aimed to identify and propose an effective technique for earlier prediction of the diabetes. The data mining techniques compared in this works were namely: Artificial Neural Network, C4.5 Classifier, Support Vector Machine and K-Nearest Neighbour. The result showed that one with the highest accuracy above $89 \%$ was Artificial Neural Network.

\section{METHODOLOGY}

Four frequently used major techniques have been found in the above literature review and those are sufficient enough for data analysis and prediction of type II diabetes which are discussed in the following subsections -

\subsection{Artificial Neural Network}

The artificial neural network is as similar as natural neural network of a human brain. Artificial Neural Network mainly has three layers, they are;

Input layer: Input neurons define all the input attribute values for the data mining model and their probabilities [26].

Hidden layer: Hidden neurons receive inputs from input neurons and provide outputs to output neurons. The hidden layer is where the various probabilities of the inputs are assigned weights. A weight describes the relevance or importance of a particular input to the hidden neuron. The neuron with greater weight is assigned to an input. The value of that input is more important weights can be negative, which means that the input can inhibit rather than favour a specific result [26]. 
Forestalling Growth Rate in Type II Diabetic Patients using Data Mining and Artificial Neural Networks: An Intense Survey

Output layer: Output neurons represent predictable attribute values for the data mining model [26].

\subsection{C4.5 Algorithm}

C4.5 is a decision tree algorithm. The performance of C4.5 is recursively separate assessment in branches to build tree for the reason of improving the calculation accuracy. Systems that construct classifiers are one of the commonly used data mining tools. Such systems take a collection of cases as input, each case belonging to one of a small number of classes that is described by its values for a fixed set of attributes, and output a classifier that can accurately predict the class to which a new case belongs [26].

\section{3. $K$-nearest neighbors Algorithm}

K-nearest neighbors is a simple algorithm that stores all available cases and classifies new cases based on a similarity measure. The neighbors are taken from a set of objects for which the object property value (for k-NN regression) or the class (for k-NN classification) are recognized. This can be supposed of as the training set for the algorithm, though no explicit training step is essential. Significance of $\mathrm{k}$ is constantly a positive integer. Here neighbors are selected from set of objects so constantly accurate classification is recognized. Categorizing objects based on the following exercise data in the feature space [22].

Following are steps used to perform prediction through KNN algorithm.

Step 1: Define k means the magnitude of nearest neighbors.

Step 2: Calculate the distance among the query instance and all the training samples.

Step 3: The distance of entire the training samples are organized and nearest neighbor constructed on the $\mathrm{k}$ minimum distance is resolute.

Step 4: Acquire entire the classes of the training data for the structured value which falls under $\mathrm{k}$.

Step 5: Utilize simple majority of group of nearest neighbors as the forecast value of the query instance.

\subsection{Support Vector Machines (SVMs)}

The support vector machine is a training algorithm for learning classification and regression rules from data. The SVM is based on statistical learning theory. There are two ways of implementing SVM. The first technique involves mathematical programming and the second technique employs kernel functions. When kernel functions are used, SVM focuses on dividing the data into two classes, $\mathrm{P}$ and $\mathrm{N}$, corresponding to the case when $\mathrm{yi}=+1$ and $\mathrm{yi}=-$ 1 , respectively. The support vector classification searches for an optimal separating surface, called a hyper plane, which is equidistant from each of the classes. This hyper plane has many important statistical properties and kernel functions are non-linear decision surfaces [2].

\section{ANALYSIS OF REVIEWED WORKS}

The accuracy of a prediction of diabetes needs to be evaluated before it can become useful. Limited availability of data often makes estimating accuracy a difficult task. Choosing a good evaluation methodology is very important for data mining and Artificial Intelligence. There are several popular techniques used for such evaluation. The reviews of these data mining and Artificial Intelligence techniques lead us to a conclusion that the applications of ANN show very encouraging results comparing with SVM, KNN, and C4.5 similar techniques [26]. 
Since, ANN is known to be a Universal Predictor, it predicts with relatively accuracy and efficiency.

\section{RESULT AND DISCUSSION}

In this work, the Pima Indian Diabetes dataset was used for comparing various data mining algorithms with prediction accuracy. The table [26] is illustrated below.

Table 1 Performance of Different Data Mining Algorithms [26]

\begin{tabular}{|c|c|}
\hline $\begin{array}{c}\text { Algorithm } \\
\text { Used }\end{array}$ & $\begin{array}{c}\text { Predicted } \\
\text { Accuracy }\end{array}$ \\
\hline SVM & $74.8 \%$ \\
\hline KNN & $78 \%$ \\
\hline C4.5 & $86 \%$ \\
\hline ANN & $89 \%$ \\
\hline
\end{tabular}

From the above literature survey, it is observed that the ANN provides more accurate result than other classification techniques such as SVM, KNN and C4.5. The below figure shows the graphical presentation of the comparison results between different data mining algorithms.

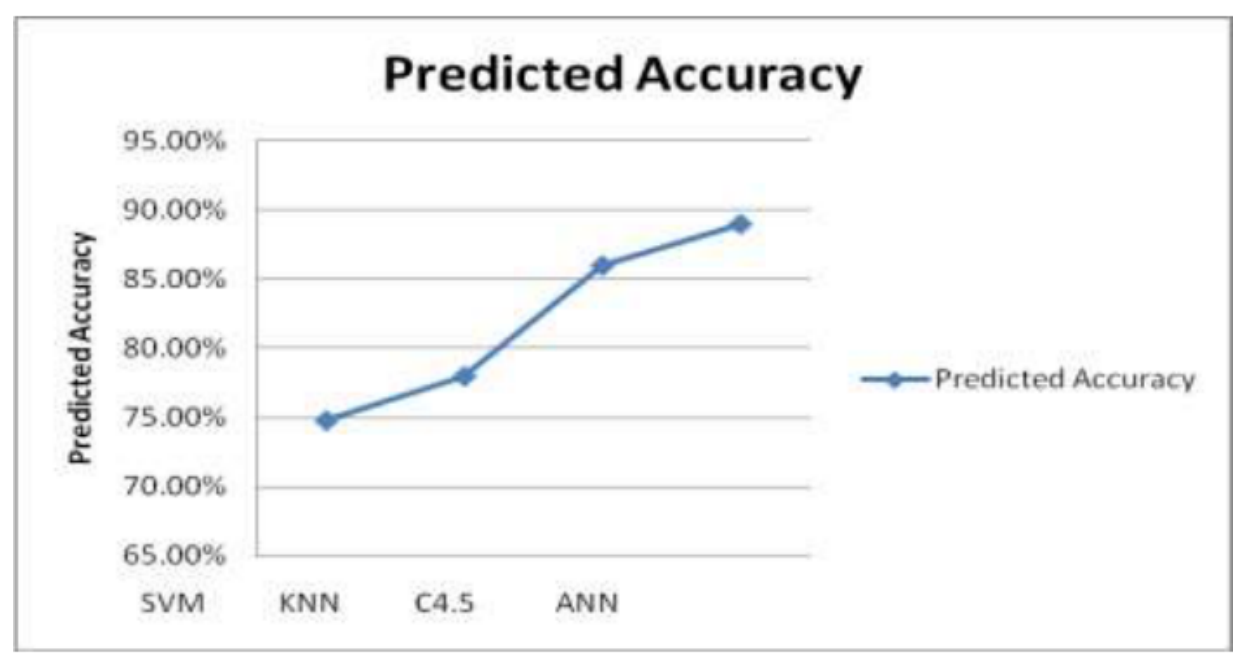

Based on the reviewed literature, the ANN is known for applying effective prediction of diabetes. This work will be implemented in my future course of experimentation in predicting the types of diabetes.

\section{CONCLUSIONS}

Data mining has played a key role in diabetes research. Data mining would be an important asset for diabetes researchers because it can discover unseen knowledge from a huge amount of diabetes-related data. I believe that data mining and Artificial Neural Network can notably help diabetes research and finally improve the quality of health care for diabetes patients. The data mining techniques compared in this work are - ANN, SVM, KNN, and C4.5. Then ANN has the highest accuracy of $89 \%$. As a universal predictor, ANN can play an essential role in various healthcare applications and predictions. 
Forestalling Growth Rate in Type II Diabetic Patients using Data Mining and Artificial Neural Networks: An Intense Survey

\section{REFERENCES}

[1] Kavitha, K., Sarojamma, S. R., Monitoring of Diabetes with Data Mining via CART Metod. International Journal of Emerging Technology and Advanced Engineering Website: www,ijetae.com , ISSN 2250-2459, Volume 2, Issue 11,2012.

[2] Aljumah, A. A., Ahamad, M. G., Siddiqui, M., K., Application of data mining: Diabetes health care in young, Journal of King Saud University - Computer and Information Sciences , 25, 2013, pp.127-136.

[3] Singh, S., K. and Archana, A data mining approach for the diagnosis of diabetes mellitus, Intelligent Systems and Control (ISCO), 7th International Conference, Coimbatore, India: IEEE, 2013.

[4] Rahman, R. M., Afroz, F., Comparison of Various Classification Techniques Using Different Data Mining Tools for Diabetes Diagnosis, Journal of Software Engineering and Applications , 6, 2013, pp. 85-97.

[5] Rajesh, K., Sangeetha, V., Application of Data Mining Methods and Techniques for Diabetes Diagnosis, International Journal of Engineering and Innovative Technology (IJEIT), Volume 2, Issue 3, 2012.

[6] Iyer, A., Jeyalatha, S., Sumbaly, S., Diagnosis of Diabetes Using Classification Mining Techniques, International Journal of Data Mining \& Knowledge Management Process (IJDKP), Vol.5, No.1, 2015.

[7] Thirumal, N., and Nagarajan, Utilization of Data Mining Techniques for Diagnosis of Diabetes Mellitus - A Case Study ,ARPN Journal of Engineering and Applied Sciences, VOL. 10, NO. $1,2015$.

[8] Barakat, Nahla, Andrew P. Bradley and Nabil, M., Barakat, H., Intelligible Support Vector Machines for Diagnosis of Diabetes Mellitus, IEEE Transactions on Information Technology in Biomedicine, 2010, pp. 1114-1120.

[9] Kumari, M.,Vohra, R. and Arora, A., Prediction of Diabetes Using Bayesian Network, (IJCSIT) International Journal of Computer Science and Information Technologies, , Vol. 5 (4), 2014, pp. 5174-5178.

[10] Priya, S., Rajalaxmi, R. R., An Improved Data Mining Model to Predict the, International Conference on Recent Trends in Computational Methods, Communication and Controls (ICON3C 2012), 2012 .

[11] Kanungo, Y. S., Srinivasan, B., Choudhary, S., Detecting Diabetic Retinopathy using Deep,2nd IEEE International Conference On Recent Trends in Electronics Information \& Communication Technology (RTEICT), India, , 2017.

[12] Rahimloo, P., Jafarian, A., Prediction of Diabetes by Using Artificial Neural Network,Logistic Regression Statistical Model and Combination of Them, Bulletin de la Société Royale des Sciences de Liège, Vol. 85, 2016, pp. 1148 - 1164.

[13] Wu, H., Yang, S., Huang, Z., He, J., Wang, X., Type 2 diabetes mellitus prediction model based on data mining. Elsevier Ltd. Beijing, 2017.

[14] Motka, R., Parmar, V., Kumar, B., Verma, A. R., Diabetes Mellitus Forecast Using Different Data Mining Techniques, 4th International Conference on Computer and Communication Technology (ICCCT), 2013 .

[15] Anjaneya, L. H., Holi, M., S., Multilayer machine learning algorithm to classify diabetic type on knee dataset, IEEE International Conference On Recent Trends In Electronics Information Communication Technology, India , 2016. 
[16] Karan, O., Bayraktar, C., Kaya, H. G., Karlık, K., Diagnosing diabetes using neural networks on small mobile devices, Elsevier Ltd. Expert Systems with Applications 39 , 2012, pp. 54-60.

[17] Jasim, I., S., Duru, A. D., Shaker, K., Abed, B., M. and Saleh, H., M., Evaluation and Measuring Classifiers of Diabetes, IEEE, ICET2017, 2017.

[18] Santhanam, T., Padmavathi, M. S., Comparison of K-Means Clustering and Statistical Outliers in Reducing Medical Datasets, International Conference on Science, Engineering and Management Research (ICSEMR 2014) , 2014.

[19] Hamdi, T., Benali, J., Fnaiech, N., Di Costanzo,V., Fnaiech, F., Moreau, E., and Ginoux, J., M., Artificial Neural Network for Blood Glucose Level Prediction, International Conference on Smart, Monitored and Controlled Cities (SM2C), Kerkennah, Tunisia, 2017.

[20] Geman, O., Chiuchisan, L. and Toderean, R., Application of Adaptive Neuro-Fuzzy Inference System for Diabetes Classification and Prediction, The 6th IEEE International Conference on E-Health and Bioengineering - EHB 2017, 2017 ,pp. 639-642.

[21] Kalaiselvi, C. and Nasira, G. M., A New Approach for Diagnosis of Diabetes and Prediction of Cancer using ANFIS, 2014 World Congress on Computing and Communication Technologies , 2014, pp. 188-190.

[22] NirmalaDevi, M., Balamurugan, A. S. and Swathi U.V., An amalgam KNN to predict Diabetes Mellitus, IEEE International Conference on Emerging Trends in Computing, Communication and Nanotechnology (ICECCN 2013), 2013, pp. 691-695.

[23] Swain, A., Mohanty, S. N. and Das, A. C., Comparative Risk Analysis on Prediction of Diabetes Mellitus Using Machine Learning Aproach, International Conference on Electrical, Electronics, and Optimization Techniques (ICEEOT) , 2016, pp. 3312-3317.

[24] Zecchin, C., Zecchin, Facchinetti, A., Sparacino, G., Nicolao, G., D., and Cobelli, C., A New Neural Network Approach for Short-Term Glucose Prediction Using Continuous Glucose Monitoring Time-Series and Meal Information, 33rd Annual International Conference of the IEEE EMBS , 2011, pp. 5653-5656.

[25] Zecchin, C., Facchinetti, A., Sparacino, G., Nicolao, G., D. and Cobelli, C., Neural Network Incorporating Meal Information Improves Accuracy of Short-Time Prediction of Glucose Concentration. IEEE Transactions on Biomedical Engineering, VOL. 59, NO. 6 , 2012, pp.1550-1560.

[26] M. Durairaj and G. Kalaiselvi, Prediction Of Diabetes Using Soft Computing TechniquesA Survey. International Journal of Science \& Technology Reasearch, Volumne 4, Issue 3, 2015, pp.190-191. 